\title{
Dimensionnement à la fatigue thermomécanique de structures dans l'industrie automobile
}

\author{
Éric Charkaluk ${ }^{1, a}$, André Bignonnet ${ }^{2}$ et Jean-Jacques Thomas ${ }^{2}$ \\ 1 Laboratoire de Mécanique de Lille, UMR CNRS 8107, boulevard Paul Langevin, 59655 Villeneuve d'Ascq Cedex, France \\ 2 P.S.A. Peugeot-Citroën, Direction de la Recherche et de l'Innovation Automobile, Centre de Vélizy, route de Gisy, \\ Bât. 57 rdc, 78943 Vélizy-Villacoublay, France
}

Reçu le 10 juillet 2002, accepté le 23 octobre 2003

\begin{abstract}
Résumé - Cet article présente une approche globale du dimensionnement à la fatigue thermomécanique des structures dans l'industrie automobile. Cette approche repose sur quatre aspects : la définition du chargement, la modélisation du comportement du matériau, celle de l'endommagement et un critère de ruine de la structure. Cette démarche est appliquée au cas de culasses et de collecteurs d'échappement soumis à des chargements thermiques transitoires. Les hypothèses et choix principaux nécessaires à un calcul intégré dans un schéma de développement industriel sont tout d'abord présentés. Deux points sont plus particulièrement développés : le comportement mécanique et l'endommagement. Deux modèles de comportement viscoplastique ont été choisis et leurs paramètres identifiés à partir d'essais isothermes et validés à l'aide d'essais anisothermes. Un critère de dimensionnement basé sur l'énergie mécanique dissipée dans le matériau est proposé. Associé à un calcul de structure, il permet une estimation de la durée de vie de pièces soumises à un chargement cyclique multiaxial anisotherme. On a ainsi accès dès la phase de conception à des calculs prédictifs de la tenue en service de composants automobiles.
\end{abstract}

Mots clés : Fatigue / conception / structure / thermomécanique / viscoplasticité / énergie

Abstract - Thermomechanical design of structures in the automotive industry. This article presents a global approach for the design of structures undergoing thermomechanical loadings in the automotive industry. This approach is based on four topics: the applied loading, the modeling of the material behaviour, the modeling of the fatigue damage and a failure criterion. This method is applied for the design of cylinder head and exhaust manifold undergoing a thermal transient loading. In order to integrate this approach in an industrial development scheme, the initial hypothesis for the modeling are first presented. Two particular points are then underlined: the mechanical behaviour and the damage modeling. Two viscoplastic constitutive laws are chosen and their parameters are calibrated by using isothermal tests and validated with non isothermal tests. A design criterion based on the mechanical energy dissipated in the material is proposed. The analysis of the computations results on structures with this criterion yields an estimation of the lifetime in a non isothermal multiaxial context. This allows to guaranty the integrity of the structure, as soon as the design exists.

Key words: Fatigue / design / structure / thermomechanics / viscoplasticity / energy

\section{Introduction}

L'étude des composants métalliques sollicités à hautes températures a réellement débuté dans les années cinquante par le biais de l'industrie aéronautique puis avec le démarrage de l'industrie nucléaire. Les métaux ont alors été soumis à des températures dépassant parfois la moitié de la température de fusion, domaine dans lequel leurs

a Auteur correspondant :

eric.charkaluk@univ-lille1.fr caractéristiques mécaniques sont considérablement modifiées et où les mécanismes d'endommagement se multiplient. Les cycles de sollicitations que les structures subissent entraînent alors fréquemment des déformations inélastiques cycliques.

Ainsi, la fatigue des métaux, longtemps étudiée dans le cadre des grands nombres de cycles et de l'endurance illimitée (fatigue polycyclique) a vu soudain son domaine d'étude s'étendre à l'endurance limitée et aux faibles nombres de cycles (fatigue oligocyclique). En 


\section{Nomenclature}

\begin{tabular}{|ll|}
\hline $\mathbf{A}$ & déviateur du tenseur des contraintes \\
$\mathbf{C}$ & tenseur des modules élastiques \\
$\mathbf{C}_{\mathbf{p}}$ & tenseur des modules élastiques de la partie élastoplastique \\
$\mathbf{C}_{\mathbf{v}}$ & tenseur des modules élastiques de la partie viscoélastique \\
$E$ & module d'élasticité \\
$H$ & module d'écrouissage \\
$J_{2}$ & second invariant du déviateur du tenseur des contraintes \\
$K_{p}$ & module d'élasticité de la partie élastoplastique \\
$K_{v}$ & module d'élasticité de la partie viscoélastique \\
$m$ & exposant de la loi de viscosité de Norton-Hoff \\
$N_{\text {sat }}$ & nombre de cycles à saturation \\
$N_{t a n}$ & nombre de cycles de fin de stabilisation \\
$N_{r}$ & nombre de cycles à rupture \\
$\varepsilon$ & tenseur des déformations mécaniques \\
$\Delta \varepsilon$ & amplitude de déformation totale \\
$\varepsilon_{e l}$ & tenseur des déformations élastiques \\
$\varepsilon_{\mathbf{i n}, \mathbf{p}, \mathbf{v}, \mathbf{v p}}$ & tenseur des déformations respectivement inélastiques, plastiques, visqueuses et viscoplastiques \\
$\gamma_{p, v, v p}$ & multiplicateur respectivement plastique, visqueux et viscoplastique \\
$\eta$ & viscosité \\
$\sigma_{\mathbf{p}, \mathbf{v}, \mathbf{v p}}$ & tenseur des contraintes respectivement plastiques, visqueuses et viscoplastiques \\
$\sigma_{m a x}$ & contrainte maximale \\
$\sigma_{u}$ & contrainte ultime \\
$\sigma_{y}$ & limite d'élasticité \\
$\Delta w$ & densité d'énergie dissipée par cycle \\
$\Delta w_{\text {sat }}$ & densité d'énergie dissipée par cycle à saturation \\
$W_{\text {sat }}$ & densité d'énergie dissipée cumulée à saturation \\
\hline
\end{tabular}

50 ans, un grand nombre d'avancées ont été réalisées dans plusieurs domaines. On peut citer entre autres la compréhension et la modélisation des phénomènes physiques mis en jeu dans les mécanismes de déformation, le développement de modèles de comportement mécanique compatibles avec des calculs de structures par éléments finis, le développement de machines d'essais à hautes températures de plus en plus performantes permettant la réalisation d'essais isothermes et anisothermes mieux contrôlés et enfin la compréhension et la modélisation des mécanismes d'endommagement à hautes températures.

En outre, la simulation numérique a profité ces 20 dernières années d'un essor des moyens de calculs associé à un développement de nouveaux algorithmes de résolution. Ceux-ci permettent aujourd'hui la réalisation de calculs de structures de plus en plus rapides.

Dans le même temps, dans l'industrie mécanique et en particulier dans l'industrie automobile, la mise en oeuvre de calculs prédictifs est devenue de plus en plus indispensable afin d'éviter la réalisation d'un trop grand nombre de prototypes, de diminuer les temps de conception et donc les coûts. Il est alors impératif que ces calculs puissent s'intégrer dans un schéma de développement en apportant les réponses souhaitées - c'est-à-dire, dans notre cas particulier, la tenue en service de la pièce estelle garantie ou non? - avant la réalisation d'un prototype physique. Nous allons illustrer ici cette démarche prédictive dans le cas de deux pièces automobiles soumises à des sollicitations de fatigue thermomécanique : la culasse et le collecteur d'échappement. Les géométries sont complexes, particulièrement dans le cas de la culasse, le chargement thermique est transitoire et la prédiction de la tenue en service est ainsi assez délicate.

Peu d'études ont été réalisées sur de telles structures soumises à des chargements thermomécaniques multiaxiaux. On peut citer, dans l'industrie automobile et en dehors des présents travaux, deux études réalisées sur ce type de problématique : il s'agit de modèles de dimensionnement à la fatigue thermomécanique en vue d'application sur les culasses issus des travaux de l'École des mines de Paris [1] d'une part et de l'University of Illinois $[2,3]$ d'autre part. Dans les deux cas, les culasses sont réalisées dans le même alliage d'aluminium AS7U3G traité T7 [1] et T6 $[2,3]$. Avant d'identifier ce qui différencie les présents travaux de ces deux études, il est important de préciser ce que l'on entend par une démarche de dimensionnement à la fatigue. Une telle démarche repose sur quatre points : le chargement, le comportement mécanique du matériau, l'endommagement sous sollicitations cycliques et la ruine de la structure. Ces quatre aspects sont liés entre eux : le chargement et le comportement du matériau apportent la réponse mécanique de la structure et celle-ci va permettre de déterminer la durée de vie de la structure par l'intermédiaire d'un indicateur de l'endommagement, durée de vie dépendant bien entendu du critère de ruine retenu. Pour chacun de ces quatre aspects, expérimentation et modélisation sont menées conjointement. L'expérimentation sert à la fois à la compréhension des phénomènes physiques en vue de la modélisation et à l'identification des grandeurs physiques associées aux modèles. Une approche en dimensionnement des structures ne peut alors se concentrer sur 
un seul des quatre aspects évoqués précédemment. Elle doit être menée de manière globale afin d'obtenir une robustesse suffisante de la réponse apportée [4].

Ainsi, dans un premier temps, nous aborderons les hypothèses et les choix qui nous semblent importants et/ou essentiels en vue de proposer une démarche globale de dimensionnement - c'est-à-dire de la modélisation du chargement à la détermination de la durée de vie. Nous pourrons alors positionner cette approche de dimensionnement au regard des autres démarches développées dans l'industrie automobile. Nous verrons ensuite l'adaptation qui en est faite dans le cas de deux structures : la culasse et le collecteur d'échappement et nous montrerons qu'il est possible de dimensionner de telles pièces à la fatigue thermomécanique dès la phase de conception en bureau d'études.

\subsection{Chargement}

L'élément principal du chargement est la thermique (le chargement mécanique est essentiellement lié au serrage des différentes pièces entre-elles et au frettage des sièges ou guides de soupape; des modélisations usuelles permettent de bien représenter ces différents aspects). C'est un point crucial de l'approche puisque la précision des données thermiques conditionne au premier ordre la précision de la réponse mécanique et donc l'estimation de la durée de vie.

Dans un premier temps, l'objectif est d'être capable de simuler numériquement un essai moteur réalisé au banc et utilisé pour la validation. Les données d'entrée sont issues d'un calcul de combustion moteur utilisant des outils spécialisés développés par ailleurs (KIVA, FIRE, WAVE, FLUENT, ...). Ces données sont d'une part utilisées comme flux thermique sur la face flamme de la culasse et sont associées d'autre part au débit de gaz dans les conduits d'échappements afin de déterminer les températures de gaz et donc les coefficients d'échange interne $\mathrm{du}$ collecteur. Un calcul thermique transitoire est réalisé dans les deux cas dans ABAQUS/Standard et permet de connaître le chargement thermique des structures en vue d'un calcul thermomécanique (pour plus de détails, [5]). La détermination du chargement thermique constitue encore aujourd'hui la principale difficulté d'une approche de dimensionnement en fatigue thermomécanique dans l'industrie automobile (en particulier pour les échanges thermiques entre les différentes pièces du moteur : culasse, bloc, collecteur, piston, ...) et nécessitera vraisemblablement encore beaucoup de travaux dans les années à venir.

Dans un second temps, il est important que le chargement thermique appliqué lors des essais au banc soit représentatif de la clientèle du point de vue de l'endommagement en fatigue. Une méthode d'analyse probabiliste de la tenue en fatigue au regard des sollicitations en clientèle appelée approche contrainte-résistance [6] est fréquemment utilisée dans le cadre de la fatigue à grand nombre de cycles. Les éléments principaux de cette méthode sont : une méthode de comptage de cycles (par exemple comptages Rainflow, dépassements de niveaux, ...), une méthode d'équivalence en fatigue (cumul de Miner, parabole de Gerber, ...), une analyse du risque et la prise en compte des dispersions (matériau, procédé, ...).

Cette méthode doit être étendue au cas de la fatigue thermique, ce qui est rendu particulièrement délicat par les fortes non linéarités (comportement, chargement, ...) et suppose donc de nombreuses hypothèses simplificatrices. Cette approche est cependant indispensable si on souhaite positionner les bancs d'essais ainsi que les roulages par rapport à un usage en clientèle. Ceci constitue également un domaine de recherche important dans l'industrie automobile.

\subsection{Comportement mécanique}

De nombreux auteurs se sont intéressés à la modélisation des phénomènes physiques associés aux mécanismes de déformation à hautes températures. On peut se référer à François et al. [7], Lemaitre et Chaboche [8] ainsi qu'à Besson et al. [9] pour tous ces aspects. Les principaux travaux s'intéressant à la modélisation mécanique du comportement des matériaux à hautes températures ont réellement débuté avec l'essor des industries nucléaire et aéronautique. On retiendra les travaux de Prager [10], Armstrong et Frederick [11] ou encore Bui [12] sur la modélisation de l'écrouissage, de Chaboche et Lemaitre [8] sur les lois viscoplastiques à écrouissage cinématique et isotrope, de Cailletaud [13] sur la généralisation de ces lois avec plusieurs potentiels de dissipation.

Deux approches se sont particulièrement développées à cette époque : la théorie unifiée caractérisée par des lois de comportement possédant une seule déformation inélastique et la mécanique de l'endommagement initiée par Kachanov [14] et poursuivie entre autres par Lemaitre [15] et Chaboche [16]. Ces deux aspects ont été par ailleurs couplés par Lemaitre et Chaboche [8] dans des lois de comportement plastiques et viscoplastiques prenant en compte l'endommagement continu. On peut retenir de ces travaux quelques aspects principaux. L'écrouissage est généralement décomposé en une part isotrope, qui correspond à la variation de la taille du domaine d'élasticité et qui est un scalaire souvent noté $R$, et en une part cinématique, qui correspond au déplacement du centre du domaine d'élasticité dans l'espace des contraintes et qui est un tenseur souvent noté $\mathbf{X}$. Ce cadre permet alors de prendre en compte les effets de rochet $[17,18]$ ou encore de vieillissement liés à des changements microstructuraux $[1,19]$ par l'ajout de variables internes. L'ensemble des travaux précédemment cités a montré que dans le cas général, une représentation suffisamment complète du comportement plastique ou viscoplastique de nombreux matériaux métalliques faisait appel à un écrouissage isotrope et un écrouissage cinématique, tous deux non linéaires [9]. Un 
certain nombre d'inconvénients peuvent cependant être soulignés :

- les lois de comportement peuvent rapidement devenir complexes si on veut modéliser l'ensemble des phénomènes rencontrés. En particulier, le nombre de paramètres caractéristiques devient très important et leurs couplages éventuels rend l'identification délicate;

- ces modèles mécaniques ne sont pas forcément toujours représentatifs de l'ensemble des phénomènes physiques ce qui peut expliquer qu'ils sont souvent imparfaits pour modéliser correctement et le fluage et la relaxation [7];

- le couplage entre comportement et endommagement entraîne à la fois des difficultés d'identification, d'implémentation numérique et surtout des calculs très coûteux en temps. Un article récent de Sermage et al. [20] a cependant montré que ce couplage n'était, la plupart du temps, pas nécessaire.

Pour toutes ces raisons, le choix qui a été réalisé dans le cadre de l'approche développée repose sur deux points essentiels :

- la nécessité d'un modèle de comportement simple mais cependant représentatif du comportement du matériau dans la structure. Dans ce contexte, deux hypothèses sont prises : le découplage du comportement et de l'endommagement et l'identification des paramètres de la loi de comportement sur les cycles stabilisés obtenus aux essais;

- une implémentation dans un code de calcul par éléments finis utilisant un algorithme d'intégration numérique de la loi de comportement suffisamment robuste et stable afin de permettre des grands pas d'intégration.

\subsection{Endommagement}

De nombreux paramètres ont une influence sur les mécanismes d'endommagement rencontrés au cours d'essais de fatigue. Il s'agit en particulier de la température, de l'environnement, des contraintes moyennes, des déformations moyennes, et des temps de maintien.

Afin de déterminer une durée de vie sous une sollicitation entraînant des déformations inélastiques cycliques, deux approches ont principalement été utilisées. La première fait suite aux travaux de Manson [21] et Coffin [22] et on pourrait la qualifier de paramétrique. En effet, elle fait appel à des lois de la forme suivante :

$$
N_{R}=f\left(\Delta \varepsilon, \Delta \sigma, \nu, p_{i}, \ldots\right)
$$

où $\Delta \varepsilon, \Delta \sigma, \nu, p_{i}$ désignent respectivement une amplitude de déformation, une amplitude de contrainte, une fréquence et un certain nombre d'autres paramètres. On peut alors citer par exemple les propositions de Smith et al. [23] et de Ellyin et Golos [24] intégrant l'effet de la contrainte moyenne, la première s'appuyant sur l'amplitude de déformation totale associée à la contrainte maximale et la seconde s'appuyant sur l'amplitude d'énergie dissipée associée à une énergie élastique de traction. Ces lois ont l'avantage d'être relativement simples à définir et à caractériser mais ont souvent l'inconvénient de ne pas pouvoir être facilement généralisables aux contextes multiaxiaux et anisothermes [25].

La seconde approche fait suite aux travaux de Rabotnov [26] concernant le fluage et constitue la mécanique de l'endommagement continu, largement développée par Lemaitre et Chaboche $[27,28]$. Les lois d'endommagement obtenue par cette approche ont la forme générale suivante :

$$
\dot{d}=f\left(d, \sigma, p_{i}, \ldots\right)
$$

où $d$ désigne l'endommagement scalaire, $\sigma$ le tenseur des contraintes et $p_{i}$ un certain nombre de paramètres. On peut alors citer l'application de cette approche à la rupture ductile [29] ou encore à la fatigue [30]. Dans le domaine de la fatigue à hautes températures, l'écriture de ces lois d'endommagement dans le cadre de l'interaction entre les phénomènes de fatigue et de fluage a été développée par Chaboche [31] et ces lois ont été particulièrement appliquées dans le domaine aéronautique pour le dimensionnement des aubes de turbine. Schématiquement, on considère que l'endommagement par fluage est plutôt associé à un endommagement intergranulaire dépendant explicitement du temps alors que l'endommagement par fatigue correspond à une microfissuration transgranulaire dépendant des cycles de fatigue. La forme générale de cette loi d'interaction en fatigue-fluage proposée par Chaboche est la suivante :

$\mathrm{d} D=f_{\text {fluage }}(\sigma, T, D) \mathrm{d} t+f_{\text {fatigue }}\left(\sigma_{\text {max }}, \sigma_{\text {moy }}, T, D\right) \mathrm{d} N$

où $\sigma_{\max }$ et $\sigma_{\text {moy }}$ désignent respectivement les contraintes maximales et moyennes obtenues au cours d'un cycle. Comme on le voit, ces lois sont écrites en fonction du tenseur des contraintes. Or, la définition de la contrainte moyenne ou de la contrainte maximale et plus généralement l'utilisation de la notion de contrainte est assez délicate à température variable [32]. En effet, cela nécessite alors d'utiliser soit une contrainte réduite $\sigma / \sigma_{u}$ où $\sigma_{u}$ désigne la contrainte de rupture en traction [8], soit une température moyenne équivalente au sens de Taïra [33]. D'autre part, ces lois d'endommagement sont rapidement complexes d'écriture [34] et l'identification des paramètres (voir Sermage et al. [20] pour plus de détails) n'est plus très aisée.

Pour cette raison, il paraît intéressant dans le contexte automobile de se tourner vers les approches paramétriques en essayant de mettre en évidence les grandeurs mécaniques caractéristiques de l'endommagement à hautes températures.

\subsection{Critère de ruine}

Dans le cas de la fatigue, il est important de définir le critère de ruine adopté. De nombreuses approches s'intéressent à des phénomènes d'amorçage ou de propagation de fissures. Dans des applications automobiles, 
on doit s'intéresser à la ruine de la pièce d'un point de vue fonctionnel (Bignonnet [35]). L'objectif est d'éviter la présence d'une fissure macroscopique provoquant un disfonctionnement (perte de rigidité, d'étanchéité, ...) pendant la durée de vie de la pièce. Ainsi, tant que l'influence d'une fissure reste confinée à un élément de volume mésoscopique, elle est gouvernée par les champs mécaniques locaux et n'a pas d'effet sur le comportement mécanique global de la structure. Lorsque sa taille dépasse celle de cet élément de volume, elle est alors gouvernée par les champs mécaniques à l'échelle de la structure. Cette situation conduit rapidement à un disfonctionnement de la pièce. Ceci correspond à la définition de la ruine que nous adoptons.

Dans ce contexte, nous ne séparons pas amorçage et propagation d'une fissure et nous nous intéressons uniquement à cette notion de ruine de la structure, que ce soit pour une éprouvette, une culasse ou un collecteur d'échappement.

Sous toutes ces hypothèses, une approche globale de dimensionnement compatible avec des schémas de conception peut être envisagée. Elle va reposer maintenant sur le choix d'un modèle de comportement et d'une loi d'endommagement.

\section{Modélisation mécanique du comportement du matériau dans la structure}

La modélisation mécanique du comportement du matériau doit être réalisée en vue du calcul de structure. Il ne s'agit pas de représenter le comportement du matériau mais son comportement dans la structure, c'est-à-dire dans son contexte d'utilisation; vitesses de sollicitations, températures, environnement doivent être représentatifs de cette utilisation. Les éprouvettes utilisées pour les essais doivent être, quant à elles, représentatives de la microstructure, de la santé matière ou encore du procédé d'obtention correspondant aux pièces réelles, ce qui est particulièrement délicat - mais néanmoins nécessaire dans le cas des matériaux issus d'un procédé de fonderie. C'est un point crucial de la caractérisation et de la modélisation.

\subsection{Matériaux}

Les matériaux étudiés sont :

- une fonte à graphite sphéroïdal contenant du silicium et du molybdène et dénommée SiMo dans le cas des collecteurs d'échappement. Sa composition est donnée dans le tableau 1 ;

- un alliage d'aluminium AS7G0,3 traité T7, dans le cas des culasses. Sa composition est donnée dans le tableau 2.

Afin de stabiliser les caractéristiques mécaniques de l'alliage d'aluminium, la culasse subit un traitement thermique T7 après la coulée. Ce traitement consiste en une
Tableau 1. Composition en masse de la fonte SiMo.

\begin{tabular}{cccccc}
\hline élément & $\mathrm{C}$ & $\mathrm{Si}$ & $\mathrm{Mo}$ & $\mathrm{Mg}$ & $\mathrm{Ni}$ \\
\hline$\%$ masse & 3.47 & 3.86 & 0.63 & 0.22 & 0.56 \\
\hline
\end{tabular}

Tableau 2. Composition en masse de l'alliage d'aluminium.

\begin{tabular}{ccccc}
\hline élément & $\mathrm{Si}$ & $\mathrm{Cu}$ & $\mathrm{Mn}$ & $\mathrm{Mg}$ \\
\hline$\%$ masse & 7 & $\leq 0.01$ & $\leq 0.03$ & 0.3 \\
\hline
\end{tabular}

homogénéisation à $540^{\circ} \mathrm{C}$ afin de dissoudre les précipités, une trempe et enfin un revenu à $200{ }^{\circ} \mathrm{C}$ durant lequel les précipités cohérents Mg2Si sont formés.

\subsection{Stratégie expérimentale}

La première étape consiste à établir une stratégie expérimentale afin de pouvoir caractériser le matériau, choisir un modèle de comportement, en identifier les paramètres, les valider dans un contexte uniaxial puis dans un contexte multiaxial proche de celui de la structure.

\subsubsection{Essais isothermes uniaxiaux}

Dans notre cas, ces essais sont de deux types : tractionrelaxation-recouvrance (TRR) et fatigue oligocyclique.

Les essais TRR sont effectués à différentes vitesses de déformation et à différentes températures afin de couvrir une gamme de sollicitations représentative du contexte pièce. Ils se décomposent en une phase de traction à vitesse de déformation constante, un maintien à déformation maximale pendant un temps $t_{\text {maintien }}$ et un retour à déformation nulle. Ces cycles sont répétés jusqu'à l'obtention d'un cycle (pseudo)stabilisé. Ces essais permettent de mettre en particulier en évidence les effets de la viscosité. Les essais de fatigue oligocyclique sont réalisés dans la même gamme de température que les essais TRR et à amplitude de déformation imposée. Ils vont mettre en évidence le comportement cyclique du matériau (consolidation-déconsolidation, stabilisation ou non de la réponse mécanique, ...). Les conditions d'essais utilisées pour la fonte GS SiMo et l'AS7G0,3 sont détaillées dans le paragraphe 4 .

Ces essais isothermes sollicitent à température constante un élément de volume représentatif du matériau. Ils permettent de choisir le modèle de comportement adéquat et d'identifier les paramètres matériau associés à chaque température. Cependant le passage d'une démarche uniaxiale isotherme sur éprouvette à une démarche multiaxiale anisotherme sur structure n'est pas immédiate et peut poser un certain nombre de difficultés. Afin de découpler la multiaxialité et l'aspect anisotherme du chargement, des essais uniaxiaux anisothermes sont nécessaires. 

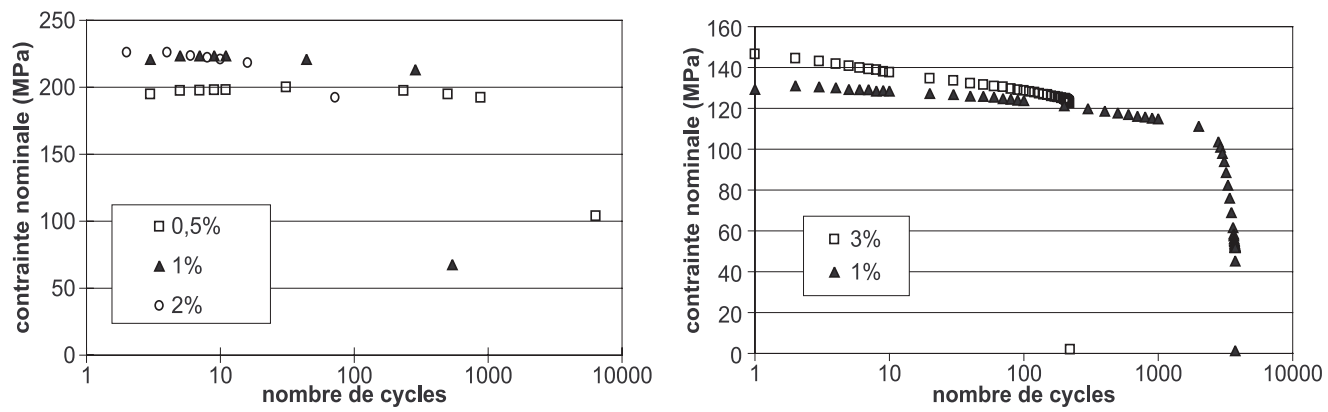

Fig. 1. Comportement cyclique de la fonte SiMo à 600 degrés (à gauche) et de l'alliage d'aluminium, vieilli, à 200 degrés (à droite). Les essais sont réalisés à amplitude de déformation imposée.

\subsubsection{Essais anisothermes uniaxiaux}

L'objectif de ces essais est de solliciter un élément de volume mécaniquement et thermiquement de manière uniaxiale. Cela permet dans un premier temps de valider dans le cas des températures variables la démarche adoptée pour le choix du modèle de comportement et pour l'identification des paramètres associés, i.e. une identification des paramètres pour chaque température.

Les difficultés de réalisation de tels essais est essentiellement liée à la maîtrise de la sollicitation thermique permettant d'obtenir des gradients pratiquement nuls dans la partie utile de l'éprouvette. Très peu de laboratoires ont les moyens et les compétences pour la réalisation de tels essais ce qui en fait un domaine de recherche expérimentale pointu, souvent lié de près aux industries aéronautique ou nucléaire (cf. Remy [36]).

\subsubsection{Essais anisothermes sur structure élémentaire}

La démarche uniaxiale nécessite une validation dans un cadre multiaxial simple mais représentatif du contexte pièce (élément de volume soumis à des champs thermiques hétérogènes). En effet, nous ne sommes plus dans un cas où des sollicitations mécaniques et thermiques sont imposées simultanément (ce qui est le cas des essais précédents) mais avec une sollicitation cyclique uniquement thermique induisant, au travers des conditions aux limites mécaniques, de la raideur de la structure ainsi que de l'hétérogénéité des champs thermiques, une réponse mécanique multiaxiale de la structure. Pour cela, des essais de fatigue thermique sur éprouvette cylindrique avec un gradient thermique important permettent d'obtenir les conditions adéquates. Ces essais sont très proches de ceux développés par Coffin [22] dans les années 50 et on peut voir apparaître lors de maintien en température des phénomènes de fluage-relaxation particulièrement discriminants pour les modèles de comportement.

\subsection{Modélisation viscoplastique}

\subsubsection{Choix du modèle}

Les structures étudiées, fabriquées en fonte ou en alliage d'aluminium, subissent des cycles thermomécaniques entre l'ambiante et une température maximale proche de $0,5 T_{f}$ (typiquement $800{ }^{\circ} \mathrm{C}$ pour les fontes et $300{ }^{\circ} \mathrm{C}$ pour les alliages d'aluminium).

D'une part, des essais isothermes de tractionrelaxation-recouvrance réalisés sur éprouvette montrent qu'à basse température ces alliages possèdent un comportement plutôt plastique (peu de relaxation de contrainte, peu de fluage), tandis qu'à haute température le comportement devient fortement visqueux (contrainte limite en fin de relaxation quasi nulle). On observe ainsi une variation importante des caractéristiques mécaniques avec la température; par exemple la limite d'élasticité conventionnelle d'une fonte GS est divisée par trois entre 400 et $800{ }^{\circ} \mathrm{C}$. Un modèle de comportement viscoplastique est donc nécessaire pour rendre compte de ces phénomènes. Nous verrons par la suite deux modèles particuliers permettant de représenter ce comportement.

D'autre part, des essais de fatigue oligocyclique effectués à différentes températures montrent une très faible consolidation-déconsolidation cyclique avec une stabilisation du comportement (contrainte nominale en fonction du nombre de cycles) à toutes les températures après un faible nombre de cycles (cf. Fig. 1). Cet aspect est particulièrement important car il permet l'hypothèse d'un comportement indépendant de l'endommagement et nous permet également de choisir la réponse stabilisée pour l'identification des paramètres, en négligeant la part isotrope de l'écrouissage. Une première hypothèse raisonnable consiste alors à considérer une loi d'écrouissage cinématique linéaire. La figure 2 a montre que cette hypothèse est tout à fait raisonnable.

Dans le cas des culasses en alliage d'aluminium AS7G0,3 traité T7, lors des maintiens en température, l'alliage subit un phénomène de vieillissement qui se traduit entre autres par une chute le la résistance mécanique. Par exemple, la limite d'élasticité conventionnelle $R e_{0,2}$ à la température ambiante est divisée par un facteur 5 entre l'état initial et l'état vieilli [37]. Contrairement aux alliages AS7U3G T6 ou T7 [1,2] pour lesquels l'état vieilli asymptotique dépend du temps et de la température, l'état vieilli asymptotique est ici unique et ne dépend que du temps. Ceci permet de découpler le comportement et le vieillissement, contrairement aux deux autres démarches, et de ne caractériser que deux états du matériau : l'état initial et l'état vieilli. Dans ces deux états, il est possible 

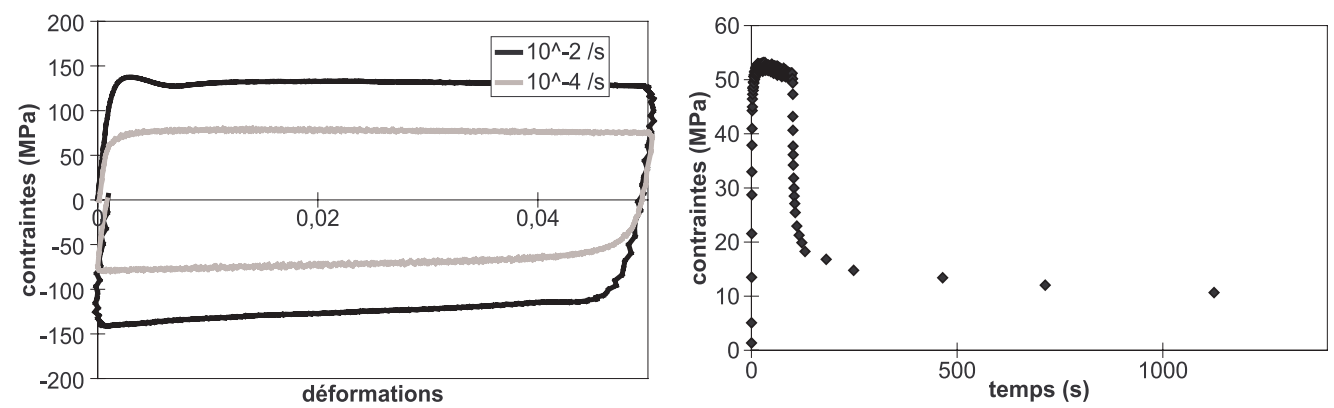

Fig. 2. Résultats typiques d'essai de traction-relaxation-recouvrance (TRR) sur la fonte. À gauche, on peut voir l'effet de la vitesse de déformation à $700{ }^{\circ} \mathrm{C}$ et à droite, la relaxation des contraintes à $800{ }^{\circ} \mathrm{C}$.
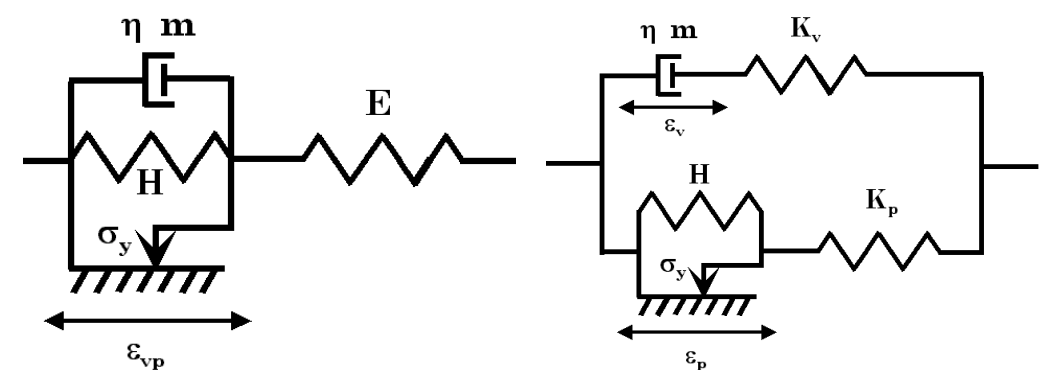

Fig. 3. Modèles rhéologiques correspondant au modèle unifié viscoplastique (à gauche) et au modèle bicouche viscoélastiqueélastoplastique (à droite).

de choisir la réponse stabilisée pour l'identification des paramètres.

Le choix du modèle de comportement va dépendre maintenant en partie de la réponse à la question suivante : est-il préférable d'avoir un comportement élastoviscoplastique ou bien un comportement viscoélastique associé à un comportement élastoplastique? La réponse est liée à la manière d'interpréter les mécanismes à l'échelle microscopique. La notion de contrainte seuil est largement justifiée pour les mouvements de dislocations (scission critique sur un plan de glissement se traduisant par une limite d'élasticité à l'échelle macroscopique). Cependant, qu'en est-il des phénomènes visqueux? Pour nombre de matériaux, les résultats d'essais de décharges partielles en fluage (voir François et al. [7]) tendent généralement à justifier l'emploi d'un seuil de viscosité conduisant alors à des modèles appelés modèles viscoplastiques unifiés. Cependant, les résultats d'essais TRR montrent que la notion de seuil disparaît progressivement à plus haute température, puisque la contrainte limite obtenue en fin de relaxation devient presque nulle. Le matériau est alors principalement visqueux. L'hypothèse d'une viscoélasticité indépendante de l'élastoplasticité n'est donc pas infondée et nous proposons alors une autre modélisation que l'on appellera modèle bicouche viscoélastique-élastoplastique.

\subsection{2 Écriture du modèle}

Il s'agit donc maintenant de détailler les deux modèles de comportement : élastoviscoplasticité à écrouissage cinématique linéaire d'une part et viscoélasticité non linéaire associée à élastoplasticité à écrouissage cinématique linéaire d'autre part. Pour les deux modèles, la fonction de charge $f$ choisie pour décrire l'inélasticité correspond au critère de von Mises :

$$
f=J_{2}(\mathbf{A})-\sigma_{y}
$$

où $J_{2}(\mathbf{A})=\sqrt{\frac{3}{2}(\mathbf{A}: \mathbf{A})}$ et $\mathbf{A}=\sigma-\mathbf{X}$ dans le cas de l'élastoviscoplasticité et de l'élastoplasticité. Le tenseur $\mathbf{X}$ correspond au déplacement de la surface de charge dans l'espace des contraintes (hypothèse d'écrouissage cinématique). C'est la variable associée au tenseur des déformations (visco)plastiques.

Dans le cas de la (visco)plasticité associée et des matériaux standards généralisés [38], on peut montrer que les lois d'écoulement inélastiques des deux modèles s'écrivent sous la forme du produit d'une norme et d'une direction exprimant la règle de normalité [9] :

$$
\dot{\varepsilon}_{\text {in }}=\gamma \cdot \frac{\mathbf{A}}{\sqrt{\mathbf{A}: \mathbf{A}}}
$$

où $\gamma \geq 0$ et $\varepsilon=\varepsilon_{\mathbf{e l}}+\varepsilon_{\mathbf{i n}}$ est la partition de la déformation totale en partie élastique et inélastique. La viscosité, enfin, est associée à une loi en puissance de type NortonHoff :

$$
\dot{\varepsilon}_{v}=\left(\frac{\sigma}{\eta}\right)^{m}
$$

Pour le modèle viscoplastique unifié présenté sur la figure $3 \mathrm{a}$, la déformation inélastique notée $\varepsilon_{\mathbf{v p}}$ est définie 

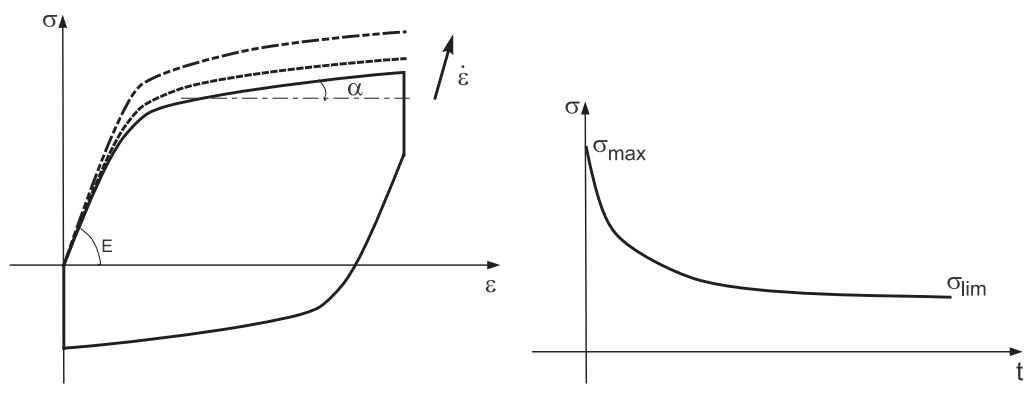

Fig. 4. Schématisation des courbes issues du cycle stabilisé d'un essai de traction-relaxation-recouvrance (TRR) telles que celles présentées sur la figure 2. Ces courbes sont utilisées pour l'identification des paramètres des modèles de comportement.

par l'équation (1) avec :

$\mathbf{A}=\operatorname{dev}(\sigma)-\frac{2}{3} H \cdot \varepsilon_{\mathbf{v p}} ; \quad \gamma_{v p}=\sqrt{\frac{3}{2}}\left\langle\frac{J_{2}(\mathbf{A})-\sigma_{y}}{\eta}\right\rangle^{m}$

Le tenseur des contraintes est lié à la partie élastique de la déformation par une loi d'élasticité $\sigma=\mathbf{C}$ : $\left(\varepsilon-\varepsilon_{\mathbf{v p}}\right)$ avec $\mathbf{C}$ le tenseur des modules élastiques (noté $E$ dans le cas unidimensionnel).

Pour le modèle bicouche, l'état interne est défini par deux déformations inélastiques, $\varepsilon_{\mathbf{p}}$ associée à la partie élastoplastique et $\varepsilon_{\mathbf{v}}$ associée à la partie viscoélastique. Comme le montre le modèle rhéologique de la figure $3 \mathrm{~b}$, le tenseur des contraintes s'écrit $\sigma=\sigma_{\mathbf{p}}+\sigma_{\mathbf{v}}=\mathbf{C}_{\mathbf{p}}$ : $\left(\varepsilon-\varepsilon_{\mathbf{p}}\right)+\mathbf{C}_{\mathbf{v}}:\left(\varepsilon-\varepsilon_{\mathbf{v}}\right)$ somme des contributions du comportement élastique de chacune des branches; $\mathbf{C}_{\mathbf{p}}$ et $\mathbf{C}_{\mathbf{v}}$ sont les tenseurs des modules élastiques de chaque partie du modèle (notés respectivement $K_{p}$ et $K_{v}$ dans le cas unidimensionnel). Les termes de la loi d'écoulement s'écrivent alors respectivement :

$$
\left\{\begin{array} { l } 
{ \mathbf { A } _ { \mathbf { p } } = \operatorname { d e v } ( \sigma _ { \mathbf { p } } ) - \frac { 2 } { 3 } H \cdot \varepsilon _ { \mathbf { p } } } \\
{ \gamma _ { p } }
\end{array} \left\{\begin{array}{l}
\mathbf{A}_{\mathbf{v}}=\operatorname{dev}\left(\sigma_{\mathbf{v}}\right) \\
\gamma_{v}=\sqrt{\frac{3}{2}}\left(\frac{J_{2}\left(\mathbf{A}_{\mathbf{v}}\right)}{\eta}\right)^{m}
\end{array}\right.\right.
$$

où le multiplicateur plastique $\gamma_{p}$ est défini par la condition de cohérence $\dot{f}=0$ définissant l'écoulement plastique.

Les deux modèles de comportement viscoplastiques retenus possèdent ainsi respectivement 5 et 6 paramètres dépendants de la température qui sont identifiés à partir du cycle stabilisé obtenu lors des essais TRR.

\subsubsection{Identification et implémentation numérique}

La première étape, après avoir choisi ces modèles, consiste à en identifier les paramètres. La méthode généralement utilisée consiste à identifier les paramètres température par température. Plusieurs démarches sont possibles : identification paramètre par paramètre dans la mesure du possible (ou plus fréquemment groupe de paramètres par groupe de paramètres en isolant ainsi paramètres plastiques et visqueux) ou encore identification de l'ensemble des paramètres simultanément, en utilisant principalement des outils d'optimisation comme SiDoLo.
Dans le cadre de cette étude, la première démarche a été retenue. Elle s'appuie sur deux types d'information présentées sur les figures 2 et 4 : la boucle d'hystérésis stabilisée (contrainte axiale-déformation axiale) des essais de traction-relaxation-recouvrance et la phase de relaxation associée dans un diagramme contrainte axiale-temps.

La démarche d'identification est similaire pour les deux modèles de comportement. Arrêtons-nous sur le modèle viscoplastique unifié. On constate sur la figure 4a que l'on peut aisément identifier le module élastique $E$ ainsi que le module d'écrouissage $\alpha$ où $\alpha=3 / 2 \cdot H$, dans l'hypothèse d'une loi d'écrouissage linéaire. Il reste alors à identifier la contrainte seuil $\sigma_{y}$ et les paramètres visqueux $\eta$ et $m$. Pour cela, comme il est montré dans [39], on peut exprimer d'une part $\sigma_{\text {lim }}$, la contrainte obtenue en fin de relaxation en fonction de $\sigma_{\max }$, définie sur la figure $4 \mathrm{~b}, \sigma_{y}, \eta$ et $m$ et on peut exprimer d'autre part la contrainte $\sigma_{v}=\sigma_{\max }-\sigma_{\text {lim }}$ (voir également Fig. 4b) en fonction de $\eta, m, E, \alpha$ et $\dot{\varepsilon}_{0}$, la vitesse de déformation constante appliquée lors de l'essai TRR. La technique adoptée correspond alors à la minimisation d'une fonction coût définie à partir de la différence entre contraintes expérimentales et calculées. Une technique simple de quadrillage peut être employée et elle revient à définir un domaine de minimisation dans $\mathbf{R}^{3}$ pour $m, \eta$ et $\sigma_{y}$.

Cependant, les paramètres identifiés température par température peuvent amener des réponses anisothermes peu conformes à celles obtenues lors d'essais sur éprouvette. La figure 5a illustre bien cette difficulté. Elle correspond à la simulation des essais de fatigue thermique sur éprouvettes soumise à un gradient thermique (cf. essais anisothermes sur structure élémentaire, paragraphes 2.2 .3 et 4 ). Cela est lié à l'existence de vallées de minima entraînant des valeurs quasi identiques de la fonction coût choisie pour des combinaisons de paramètres très différentes. Le passage de paramètre en paramètre lors de sollicitations anisothermes se fait alors différemment et les réponses mécaniques peuvent être dans ce cas très différentes d'un jeu de paramètres à un autre. Une première solution consiste à essayer le maximum de jeu de paramètres possibles jusqu'à obtenir ceux qui fourniront la meilleure réponse anisotherme; cette technique est cependant très coûteuse en temps et pas forcément très robuste. Une autre technique possible afin d'éviter ces difficultés consiste à intégrer les essais anisothermes dans l'identification des paramètres 

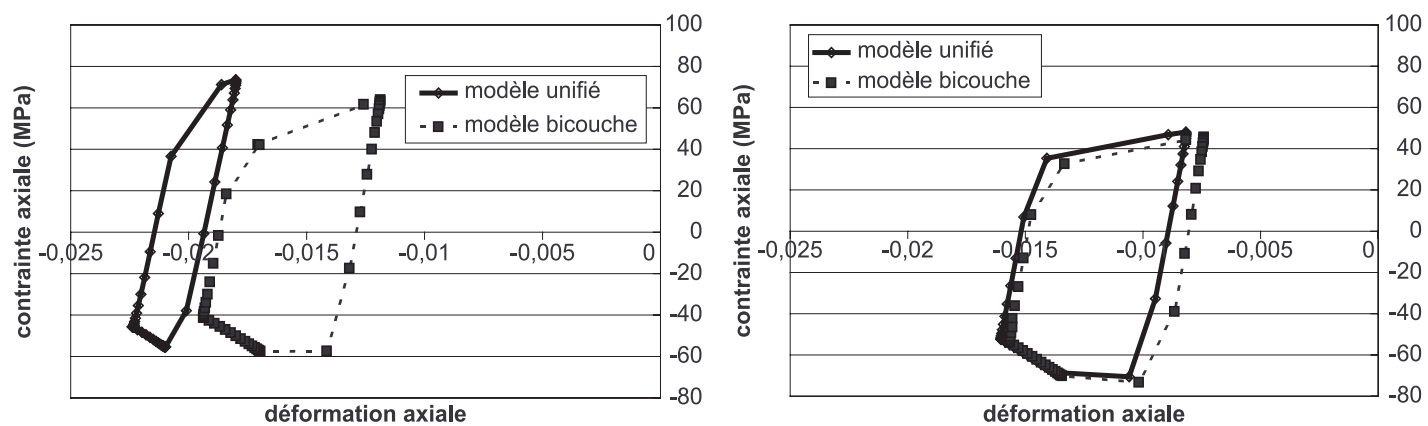

Fig. 5. Comparaison des deux modèles avec une identification isotherme (à gauche) et avec une identification isotherme et anisotherme (à droite).

et requiert alors des méthodes d'identification plus complexes. Une des méthodes possibles a été proposée par Verger et al. [37,40]. Elle est fondée sur le contrôle optimal et la méthode de l'état adjoint [41]. Elle permet alors de tenir compte facilement de plusieurs types d'essais simultanément en pondérant les fonctions coûts et la minimisation est réalisée à l'aide d'un algorithme de descente du type BFGS. Ce type d'approche peut permettre de déterminer de manière unique un ensemble de jeux de paramètres défini directement sur toute la gamme de température. La figure $5 \mathrm{~b}$ de droite montre alors que quelque soit le modèle de comportement choisi, la réponse mécanique obtenue est quasiment la même (aux différences de formulation de modèle près). Ce problème d'identification est un des points importants de l'approche de dimensionnement car il conditionne directement la validité de la réponse mécanique obtenue sur la structure.

Pour implémenter chacun des modèles dans le code de calcul ABAQUS/Standard, un schéma implicite utilisant la technique du retour radial proposé par Simo et al. [42] est utilisé. Ce schéma permet d'obtenir une convergence rapide et une réponse mécanique inconditionnellement stable (voir par exemple [43] pour les détails de l'implémentation). Une fois le modèle de comportement implémenté dans le code de calcul, les simulations numériques peuvent être réalisées sur structures complexes. L'objectif de ces simulations consiste à déterminer la réponse mécanique (pseudo)stabilisée de la structure. C'est elle qui va nous servir à définir la durée de vie. Même si elles sont utilisées ici, les méthodes incrémentales classiques ne sont pas les plus performantes pour déterminer cette réponse puisqu'elles nécessitent le calcul de tous les cycles intermédiaires afin d'atteindre le cycle stabilisé. Des études sont actuellement en cours afin de déterminer directement cette réponse stabilisée [44].

\section{Estimation des durées de vie}

\section{1 État de l'art}

L'objectif final est maintenant d'évaluer le nombre de cycles correspondant à la ruine de la structure à partir de la réponse stabilisée de celle-ci, afin d'être capable de garantir la tenue en service de la pièce calculée. Il faut pour cela définir un critère dépendant de variables mécaniques liées aux champs de contraintes et de déformations.

Les premières études concernant des critères de tenue en fatigue oligocyclique peuvent être classées dans ce que nous avons appelé précédemment les lois paramétriques.

Une étude détaillée (Charkaluk et Constantinescu [25]) a cependant montré que la loi de MansonCoffin $[21,22]$, la méthode du Strain-Range Partitioning (SRP) [45] ou la fonction de Smith-Topper-Watson (STW) [23] exprimées initialement à partir d'essais uniaxiaux isothermes n'étaient pas adaptées au problème de la fatigue thermomécanique. Les difficultés proviennent essentiellement du caractère multiaxial et de la grande amplitude des températures subies par la structure. En effet, l'interprétation classique d'essais de fatigue oligocyclique isothermes à l'aide de la loi de Manson-Coffin montre d'une part une grande différence de durées de vie pour des essais ayant la même amplitude de déformation plastique mais réalisés à des températures différentes et d'autre part ne permet pas une généralisation claire à un cas multiaxial. Une interprétation en terme de déformation plastique cumulée telle que proposée par le SRP sur une courbe contraintes-déformations anisotherme est ainsi rendue hasardeuse [46].

D'autre part, une interprétation d'essais anisothermes à l'aide de la fonction STW (fonction STW = $\sqrt{E \cdot \sigma_{\max } \cdot \Delta \varepsilon}$ ) est difficile à partir du moment où le choix de $\sigma_{\max }$ est rendu impossible dans un cas anisotherme. On peut en effet remarquer que pour la fonte, la contrainte maximale peut varier pour une même déformation de 30 à $300 \mathrm{MPa}$ selon la température [25].

Ainsi, la principale critique des critères précédents est leur généralisation impossible à un chargement quelconque.

Dans le cas des lois de fatigue-fluage développées en particulier à l'ONERA par Chaboche [31], celles-ci ont été généralisées aux cas où les températures varient durant la sollicitation par l'utilisation d'une contrainte réduite (ratio $\sigma / \sigma_{u}$ où $\sigma_{u}$ est la contrainte ultime) utilisée pour définir une contrainte maximale ou une contrainte moyenne indépendamment de la température (voir en particulier Chaboche et Gallerneau [34]). De la même manière, la justification physique de cette contrainte réduite et son extension en régime anisotherme est difficile. C'est sans doute une des raisons pour 


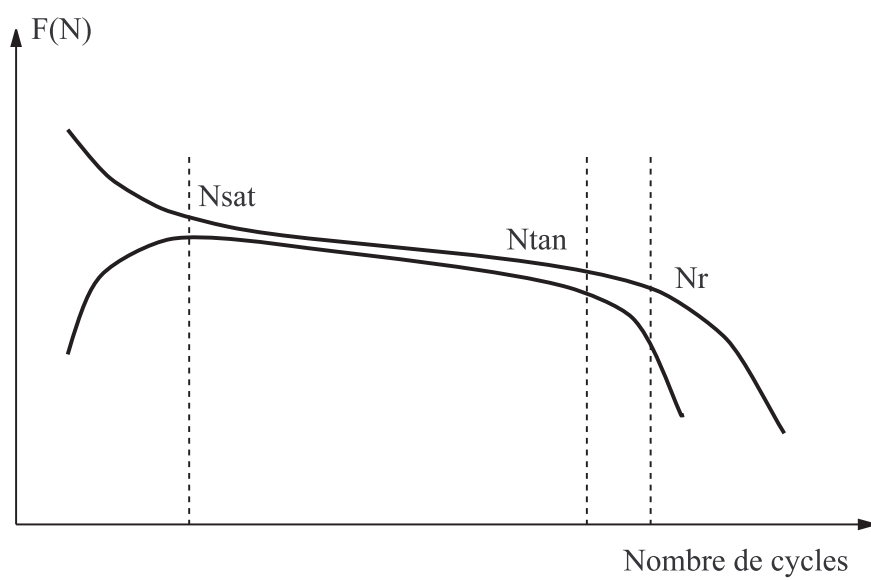

Fig. 6. Schématisation du comportement cyclique des matériaux étudiés : déconsolidation/consolidation, stabilisation, rupture.

laquelle Chaboche [32] estime qu'un travail important est nécessaire afin de redéfinir des lois d'endommagement sous un chargement en températures variables.

L'approche énergétique est une voie possible pour surmonter ces difficultés.

\subsection{Approche énergétique}

Une interprétation intéressante du comportement cyclique du matériau a été présentée par Skelton [47]. Si on observe une courbe représentant la variation de l'effort en fonction du nombre de cycles durant un essai de fatigue oligocyclique, on peut observer plusieurs phases caractéristiques (cf. Fig. 6) :

- le première phase correspond à un durcissement ou un adoucissement rapide du matériau. À la fin de cette phase, notée $N_{\text {sat }}$, le comportement mécanique est stabilisé. On peut noter $\Delta w_{\text {sat }}$ l'énergie dissipée par cycle à $N_{\text {sat }}$;

- de $N_{\text {sat }}$ à $N_{r}$, une fissure va s'amorcer et se propager. Dans le cas de nos matériaux, on peut remarquer qu'elle n'a réellement d'effet sur la structure que lorsque $N=N_{\text {tan }}$. Entre $N_{\text {sat }}$ et $N_{\text {tan }}$, la réponse mécanique est quasi-stable. À partir de $N_{\text {tan }}$, l'effort chute brusquement : c'est la ruine de l'éprouvette.

Skelton [47] remarque que l'énergie dissipée cumulée jusqu'à la réponse cyclique stabilisée du matériau, $W_{\text {sat }}=$ $\sum_{i=1}^{N_{s a t}} \Delta w$, peut être considérée comme constante pour les différents aciers étudiés. Cela lui permet de définir des lois de propagation dépendant de cette valeur $W_{\text {sat }}$, assimilée à l'énergie nécessaire à la rupture de la zone d'élaboration en pointe de fissure (process zone) (Skelton et al. [48]).

Dans ce travail on se propose plutôt d'interpréter les résultats du point de vue d'une ruine de la structure. Cette démarche est justifiée par le fait qu'on ne peut pas connaître ni suivre l'amorçage ou la propagation de fissures dans les structures étudiées. L'objectif est alors d'éviter pour des raisons fonctionnelles la présence d'une fissure macroscopique conduisant à un disfonctionnement pendant la durée de vie visée pour la pièce. Le critère de dimensionnement doit garantir cet objectif : il s'agit donc d'un critère d'amorçage. Deux données sont alors nécessaires : une variable mécanique représentative de la vie de la structure et un critère définissant la ruine de celle-ci.

Si on note $D$ la variable mécanique représentative de l'endommagement, celle-ci va devoir prendre en compte le trajet de chargement multiaxial $(\sigma, \varepsilon)$ et l'énergie dissipée par cycle nous semble être une variable intéressante car utilisable dans ce contexte multiaxial anisotherme. En effet, la densité d'énergie dissipée par cycle en un point $M(\underline{\mathbf{x}})$ est définie par :

$$
\int_{\text {cycle }} \sigma(\underline{\mathbf{x}}): \dot{\varepsilon}(\underline{\mathbf{x}}) \mathrm{d} t
$$

scalaire définissable à partir de la définition des tenseurs de contrainte et de déformation (prenant donc en compte les aspects multiaxiaux) indépendamment de la température. En utilisant simplement cette grandeur, définie au cycle stabilisée, $\Delta w_{s t a b}$, l'effet de la température est par ailleurs faible sur les durées de vie dans le cas de la fonte GS SiMo [25] et dans le cas de l'AS7G0,3 [37].

En outre, l'expérience montre que la contrainte moyenne a souvent un effet important sur la durée de vie en fatigue et la définition prise pour cette moyenne est particulièrement importante, déjà en fatigue à grand nombre de cycles [49]. À un instant $t$ et en un point $M(\underline{\mathbf{x}})$, la contrainte moyenne est définie simplement par :

$$
\sigma_{m o y}(\underline{\mathbf{x}})=\frac{1}{3} \operatorname{tr}(\sigma(\underline{\mathbf{x}}))
$$

Pour un cycle multiaxial isotherme de période $T$, la contrainte moyenne peut être définie simplement par exemple par :

$$
\sigma_{m o y}(\underline{\mathbf{x}})=\frac{1}{T} \int_{T} \frac{1}{3} \operatorname{tr}(\sigma(\underline{\mathbf{x}})) \mathrm{d} t
$$

Cependant, il ne s'agit que d'une possibilité, puisqu'une moyenne quadratique par exemple pourrait également être prise pour définition. L'extension à un contexte anisotherme pose des difficultés supplémentaires et plusieurs définitions sont actuellement à l'étude.

En résumé, on peut noter $D\left(\Delta w, \sigma_{\text {moy }}\right)(\underline{\mathbf{x}})$ une telle variable d'endommagement. Reste maintenant à définir le nombre de cycles à la ruine $N_{\text {ruine }}$. Le nombre de cycles à la ruine obtenu sur éprouvette correspond à l'amorçage d'une fissure macroscopique entraînant rapidement la rupture de l'éprouvette. Sur des structures plus complexes, ce nombre de cycles à l'amorçage d'une fissure macroscopique, $N_{\text {ruine }}$, caractérisera également la ruine de la structure. En effet, au delà de cette durée, la fissure va se propager jusqu'à être débouchante et entraîner un disfonctionnement de la pièce (fuite de gaz sur collecteur d'échappement, fuite d'eau dans la chambre 

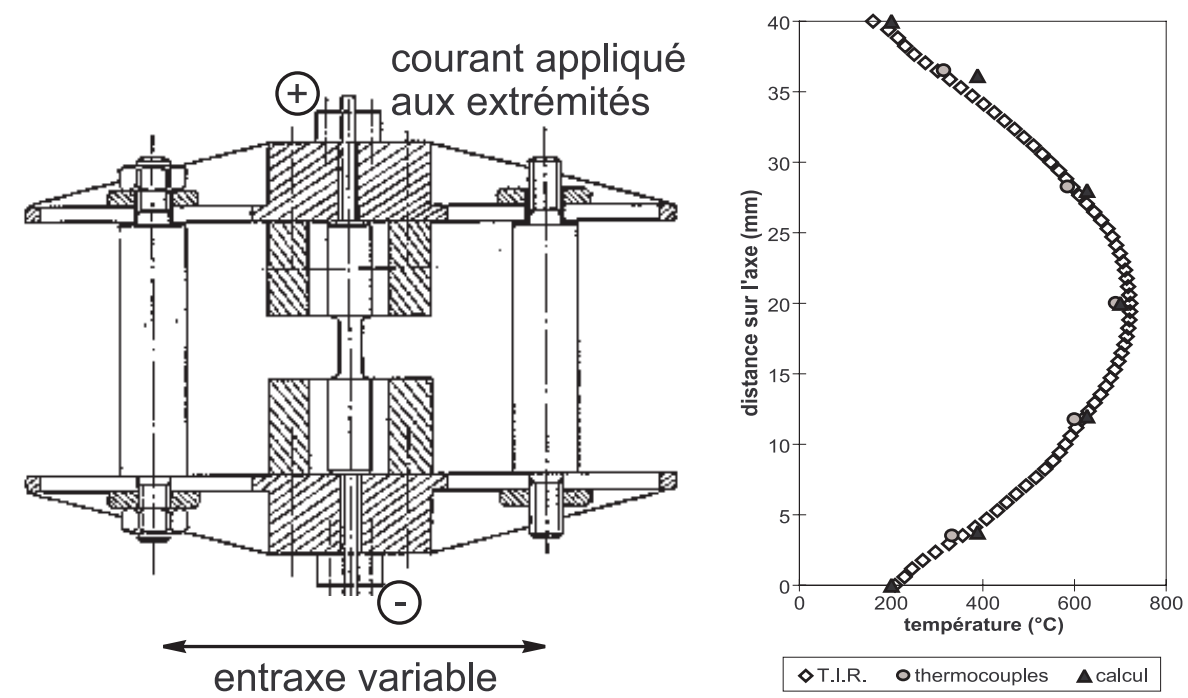

Fig. 7. Essai de fatigue thermique sur éprouvette bridée. Le chauffage est effectué par effet Joule. L'entraxe est variable et permet ainsi de sévériser plus ou moins les conditions aux limites mécaniques.

de combustion sur culasse, ...). La difficulté va donc consister en la détection de cet amorçage lors d'essais complexes et différentes techniques peuvent être utilisées (essais interrompus, suivi de l'endommagement sur structure élémentaire, détection de l'amorçage par technique optique d'analyse d'images, ...).

On peut ainsi utiliser $N_{\text {ruine }}$ et $D$ afin de caractériser l'endommagement de la structure et la courbe de dimensionnement $N_{\text {ruine }}=f(D)$ permet alors de déterminer à l'issue du calcul la durée de vie de la pièce. Dans un premier temps, nous avons utilisé dans les cas pratiques une version du critère sans prise en compte de la contrainte moyenne, noté $D_{s m}$, qui donne déjà de bons résultats en terme de prédiction de la tenue en service. Dans ce cas, $D_{s m}$ vaut simplement :

$$
D_{s m}=\Delta w_{s t a b}
$$

Les dernières validation avec prise en compte de la contrainte moyenne sont actuellement en cours.

\section{Cas pratiques : collecteurs d'échappement et culasses}

Quatre types d'essais ont été réalisés sur éprouvettes et sur pièces. Des essais isothermes pilotés en déformation ont été réalisés afin de caractériser le comportement mécanique du matériau. Les essais ont été menés à différentes températures comprises entre 20 et $300{ }^{\circ} \mathrm{C}$ ou $800{ }^{\circ} \mathrm{C}$ (respectivement pour l'AS7G0,3 et la fonte GS SiMo). Les cycles de traction-relaxation-recouvrance (TRR) présentés dans le paragraphe 2.2.1 ont été effectués à des vitesses de déformations comprises entre $10^{-4} \mathrm{~s}^{-1}$ et $10^{-2} \mathrm{~s}^{-1}$ avec des déformations maximales comprises entre 2 et $5 \%$. Les temps de maintien $t_{\text {maintien }}$ étaient de l'ordre de $30 \mathrm{~min}$. Ces essais ont permis d'identifier les paramètres des modèles viscoplastiques utilisés pour les

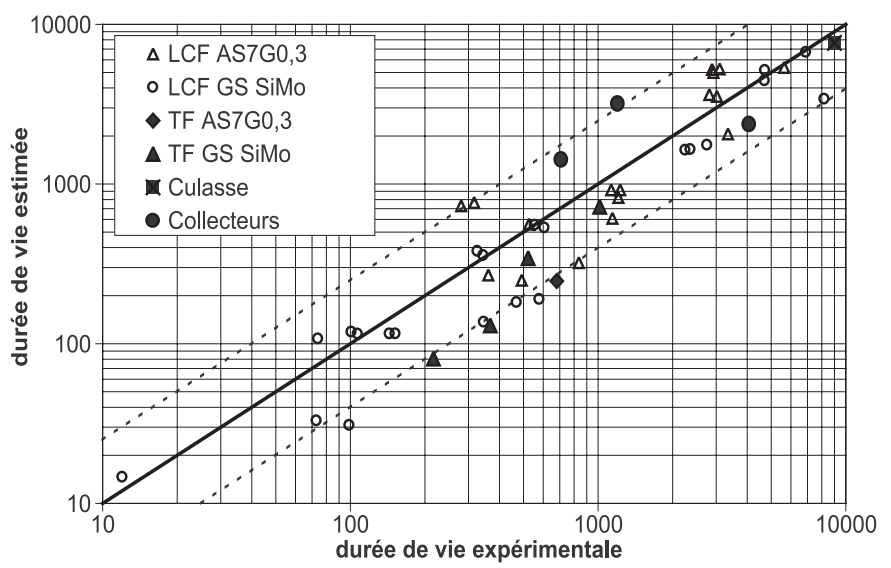

Fig. 8. Résultats obtenus pour différents types d'essais (éprouvette et structure) pour la fonte GS SiMo et l'alliage d'aluminium AS7G0,3. On remarque que l'ensemble des points est compris dans une bande de dispersion de durées de vie de plus ou moins 2,5 .

simulations de l'essai de fatigue thermique et des essais de fatigue thermomécanique sur pièce réelle.

Des essais de fatigue oligocyclique isothermes (LCF sur la Fig. 8) ont été réalisés sous un contrôle en déformation à déformation moyenne nulle $\left(R_{\varepsilon}=-1\right)$. Les essais ont été menés à différentes températures comprises entre 100 et $250{ }^{\circ} \mathrm{C}$ ou $700{ }^{\circ} \mathrm{C}$ (respectivement pour l'AS7G0,3 et la fonte GS SiMo), avec une vitesse de déformation égale à $10^{-3} \mathrm{~s}^{-1}$ et différents niveaux de déformation compris entre $\pm 0,25$ et $\pm 1,5 \%$. Les essais sont menés jusqu'à la rupture de l'éprouvette. Une analyse des courbes cycliques telles que celles présentées sur la figure 1 permet alors de déterminer le nombre de cycles à la ruine $N_{r}$. Celui-ci est défini par rapport à une chute de $10 \%$ de l'effort obtenu à $N_{t a n}$.

Des essais de fatigue thermique (TF sur la Fig. 8) ont été menés sur des éprouvettes bridées chauffées par effet 

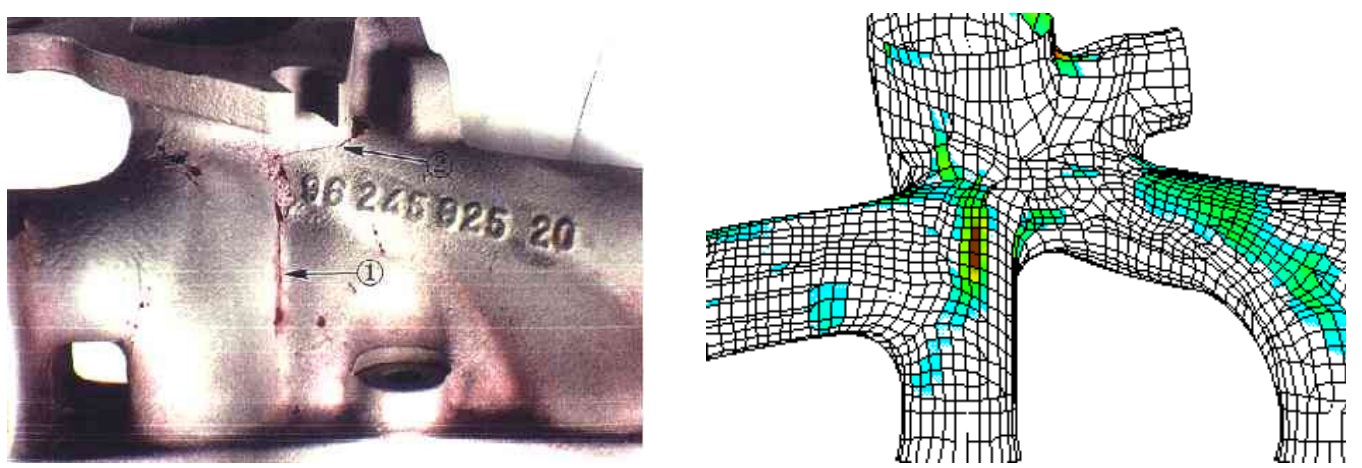

Fig. 9. Collecteur fissuré après essai et prédiction de la zone de fissuration sur la paroi interne du collecteur.

Joule (voir la présentation du montage sur la Fig. 7a). La température maximale varie de 40 à $300{ }^{\circ} \mathrm{C}$ ou $700{ }^{\circ} \mathrm{C}$ (respectivement pour l'AS7G0,3 et la fonte GS SiMo) avec une vitesse de chauffage de $20{ }^{\circ} \mathrm{C} \cdot \mathrm{S}^{-1}$. La température maximale est atteinte dans une région d'approximativement $10 \mathrm{~mm}$ au centre de l'éprouvette. Le gradient maximal est de $30-40{ }^{\circ} \mathrm{C} \cdot \mathrm{mm}^{-1}$ comme on peut le constater sur la figure $7 \mathrm{~b}$. Les paramètres d'essai sont la valeur de la raideur du montage et le temps de maintien à la température maximale. Ces essais sont menés jusqu'à rupture comme les essais de fatigue oligocyclique. Ils sont particulièrement intéressants car contrairement aux essais de fatigue oligocyclique, la déformation mécanique n'est pas imposée mais est induite par le chargement thermique. En ce sens, ils sont tout à fait représentatifs de la sollicitation subie par les collecteurs d'échappement ou les culasses.

Enfin, des essais thermomécaniques ont été réalisés sur des prototypes de collecteurs d'échappement et de culasses. Ces essais correspondent soit à des essais d'endurance sur un banc d'essai moteur, soit à des essais spécifiques sur un banc dédié à la validation des collecteurs d'échappement. Dans les deux cas, les cycles thermiques appliqués correspondent à des conditions sévères de démarrage-arrêt du véhicule (l'essai moteur est par exemple réalisé à puissance et à charge maximales). La température maximale atteinte varie alors entre 20 et $240{ }^{\circ} \mathrm{C}$ ou $700{ }^{\circ} \mathrm{C}$ (respectivement pour l'AS7G0,3 et la fonte GS SiMo). Il est important de remarquer que la température n'est pas distribuée uniformément dans la pièce et que certaines zones ne dépassent jamais 100 à $200{ }^{\circ} \mathrm{C}$ comme par exemple la majeure partie de la culasse (seule la face flamme se trouvant face au piston est fortement sollicitée thermiquement) ou la bride du collecteur d'échappement. La vitesse de chauffage est d'environ $10{ }^{\circ} \mathrm{C} \cdot \mathrm{s}^{-1}$. Ces essais présentent l'intérêt d'obtenir sur la pièce un chargement tout à fait représentatif. Les résultats des calculs simulant numériquement ces essais ont alors montré la multiaxialité des champs mécaniques locaux [4].

La première étape de la démarche de dimensionnement, en vue de sa validation, correspond à la simulation numérique des essais réalisés. Les essais de fatigue thermique sur éprouvette et les essais thermomécaniques sur prototypes ont été simulés numériquement entre autres à l'aide du code de calcul ABAQUS/Standard (pour plus de détails sur le calcul thermique et le calcul thermomécanique, voir Lederer et al. [5]). Les calculs ont été menés jusqu'à l'obtention d'une réponse mécanique (pseudo)stabilisée (5 cycles ont été nécessaires dans la plupart des cas). Cet aspect différencie particulièrement cette démarche par rapport à celles développées par l'École des mines de Paris [1] et par l'University of Illinois $[2,3]$. Les découplages entre vieillissement, endommagement et comportement du matériau permettent de fonder toute l'approche de dimensionnement autour de la notion de cycle stabilisé, autrement dit d'accommodation plastique. Dans le cas des démarches développées par l'École des mines de Paris et par l'University of Illinois, les modèles de comportement utilisés, qui intègrent en particulier explicitement le vieillissement, nécessitent de déterminer toute l'histoire de la réponse mécanique de la structure (voir par exemple les résultats numériques obtenus par Nicouleau [1]). Les calculs sont donc obligatoirement plus longs dans un schéma de développement de pièces automobiles qui est de plus en plus court (36 mois dans le cas du développement du moteur 1,4l HDi de PSA Peugeot Citroën).

La dernière étape consiste maintenant à déterminer les zones fissurées ainsi que les durées de vie des éprouvettes et des prototypes. Une première version du critère de fatigue notée $D_{s m}$ (sans prise en compte de la contrainte moyenne) a été utilisée afin d'estimer les durées de vies des éprouvettes de fatigue oligocyclique (courbes d'hystérésis expérimentales), des éprouvettes de fatigue thermique, des collecteurs et des culasses (courbes d'hystérésis numériques). La figure 8 représente la comparaison entre durées de vie expérimentales et estimées pour l'ensemble des essais de fatigue oligocyclique isothermes, de fatigue thermique sur éprouvettes et de fatigue thermomécanique sur pièces réelles. D'une part, pratiquement tous les points se trouvent dans une bande de dispersion d'un facteur $\pm 2,5$, dispersion assez courante en fatigue oligocyclique. Ce critère $D_{s m}$ permet donc bien de dimensionner des structures complexes comme les collecteurs d'échappement ou les culasses. Il faut cependant noter que les points correspondant aux essais de fatigue thermique ou aux essais sur pièces réelles sont souvent à la limite de la bande de dispersion. Or ce sont ceux 
qui présentent généralement une contrainte moyenne non nulle. Ceci montre la nécessité d'aller plus loin dans la définition de la variable d'endommagement $D$. D'autre part, sur la figure 9, on peut remarquer un bon accord entre zones fissurées lors des essais et zones prédites par le calcul. Il faut souligner que dans le cas de cette pièce souvent géométriquement complexe, ces zones ne sont pas toujours ni les plus chaudes, ni celles présentant les contraintes maximales [5]. Dans le cas de la culasse, ce n'est pas la prédiction de la zone fissurée qui constitue la difficulté, puisque celle-ci correspond presque toujours au pontet inter-sièges pour lequel les températures et les déformations sont maximales, mais bien la prédiction de la durée de vie. Cette démarche de dimensionnement est utilisée avec succès depuis quelques années dans les bureaux d'étude de PSA Peugeot Citroën.

\section{Conclusion}

Dans ce travail une méthode globale de dimensionnement à la fatigue thermomécanique des structures a été présentée. Deux lois simples de comportement et un critère de fatigue thermomécanique sont proposés. En utilisant l'énergie dissipée par cycle comme indicateur du dommage, nous avons pu franchir les difficultés liées à un chargement multiaxial et à des sollicitations thermiques instationnaires. Des calculs sur différentes structures soumises à des cycles thermomécaniques ont été réalisés et la durée de vie a été estimée en utilisant ce critère énergétique. Cette démarche globale associant une modélisation du comportement et un critère de fatigue permet un dimensionnement des structures telles que des collecteurs d'échappement ou encore les culasses dès leur phase de conception avec des temps de calculs relativement courts.

Cette démarche peut encore être améliorée, d'une part par la détermination du chargement thermique en prenant en compte l'ensemble des échanges dans le moteur (bloc, culasse, collecteur, piston, ...) et en le rendant représentatif de la clientèle par des analyses statistiques adaptées; d'autre part en prenant en compte la contrainte moyenne dans le critère de fatigue, ce qui est en cours de validation actuellement. Cette démarche est transposable à toute structure soumise à de la fatigue thermomécanique. Elle nécessite de bien modéliser le comportement du matériau dans la structure et de définir un critère de ruine à l'échelle de celle-ci.

Remerciements. Lætitia Verger (PSA Peugeot Citroën) pour son travail concernant la fatigue thermomécanique des culasses et les méthodes d'identification en viscoplasticité, Ky Dang Van et Andrei Constantinescu (Laboratoire de Mécanique des Solides - École polytechnique) pour les discussions enrichissantes sur le dimensionnement des structures et Denis Bertheau et Franck Morel (Laboratoire de Mécanique et de Physique des Matériaux - ENSMA de Poitiers) pour les essais de caractérisation.

\section{Références}

[1] E. Nicouleau-Bourles, Étude expérimentale et numérique $\mathrm{du}$ vieillissement d'un alliage d'aluminium. Application aux culasses automobiles, Ph.D. thesis, spécialité : sciences et génie des matériaux, École des mines de Paris, France, 1999

[2] T. Smith, H. Maier, H. Sehitoglu, E. Fleury, J. Allison, Modelling high-temperature stress-strain behavior of cast aluminum alloys, Met. Mat. Trans. 30A (1999) 133-146

[3] H. Sehitoglu, X. Qing, T. Smith, H. Maier, J. Allison, Stress-strain response of a cast 319-t6 aluminium under thermomechanical loading, Met. Mat. Trans. 31A (2000) 139-146

[4] E. Charkaluk, A. Bignonnet, A. Constantinescu, K. Dang-Van, Fatigue design of structures under thermomechanical loadings, Fatigue Fract. Engng. Mater. Struct. 25 (12) (2002) 1199-1206

[5] G. Lederer, E. Charkaluk, L. Verger, A. Constantinescu, Numerical lifetime assessment of engine parts submitted to thermomechanical fatigue, application to exhaust manifold design, in : SAE Technical paper series, 2000-010789, 2000

[6] J.J. Thomas, G. Perroud, A. Bignonnet, D. Monnet, Fatigue design and reliability in the automotive industry, in : G. Marquis (Ed.), Fatigue Design'98 3rd International Symposium on Fatigue Design (1998), pp. $1-11$

[7] D. Francois, A. Pineau, A. Zaoui, Comportement mécanique des matériaux - Vol. II, Hermès, 1994

[8] J. Lemaitre, J.L. Chaboche, Mécanique des matériaux solides, Dunod, Paris, 1985

[9] J. Besson, G. Cailletaud, J.L. Chaboche, S. Forest, Mécanique non linéaire des matériaux, Hermès, 2001

[10] W. Prager, Problèmes de plasticité théorique, Dunod, Paris, 1958

[11] P.J. Armstrong, C.O. Frederick, A mathematical representation of the multiaxial baushinger effect, Technical Report RD/B/N731, Berkeley Nuclear Laboratories, 1966

[12] H.D. Bui, Étude de l'évolution de la frontière du domaine élastique avec écrouissage et relations de comportement élastoplastique des métaux cubiques, Ph.D. thesis, Université Paris VI, 1969

[13] G. Cailletaud, K. Saï, Study of platic/viscoplastic models with various inelastic mechanisms, Int. J. Plasticity, 11 (8) (1995) 991-1005

[14] L.M. Kachanov, Time of the rupture process under creep conditions, Izv Akad Nauk SSR, Otd Tekn Nauk 8 (1958) 26-31

[15] J. Lemaitre, A. Plumtree, Application of damage concepts to predict creep-fatigue failures, J. Engng. Mat. Tech. 101 (1979) 284-292

[16] J.L. Chaboche, C. Stoltz, Détermination des durées de vie des aubes de turbines à gaz, Revue Française de Mécanique, 52 (1974) 37-47

[17] J.L. Chaboche, On some modifications of kinematic hardening to improve the description of ratcheting effects, Int. J. Plasticity, 7 (1991) 661-678

[18] N. Ohno, J.D. Wang, Kinematic hardening rules with critical state of dynamic recovery, part I : formulation and basics features for ratcheting behaviour, Int. J. Plasticity 9 (1993) 375-390 
[19] G. Cailletaud, Modélisation mécanique d'instabilités microstructurales en viscoplasticité cyclique à température variable, Ph.D. thesis, Spécialité : mécanique, Université Paris VI, France, 1979

[20] J.P. Sermage, J. Lemaitre, R. Desmorat, Multiaxial creep-fatigue under anisothermal conditions, Fat. Fract. Engng. Mat. Struct, 23 (2000) 241-252

[21] S.S. Manson, Behaviour of materials under conditions of thermal stresses, Technical Report TN 2933, N.A.C.A., 1953

[22] L.F. Coffin, A study of the effects of cyclic thermal stresses on a ductile material, Trans. ASME, 53-A76 (1953) 931-950

[23] K.N. Smith, P. Watson, T.H. Topper, A stress-strain function for the fatigue of metals, J. Mater. 5 (4) (1970) 767-778

[24] F. Ellyin, K. Golos, Multiaxial fatigue damage criterion, J. Engng. Mat. Tech. 113, 1988

[25] E. Charkaluk, A. Constantinescu, Energetic approach in thermomechanical fatigue for silicon molybdenum castiron, Materials at High Temperatures 17 (3) (2000) 373380

[26] Y.N. Rabotnov, Creep problems in structural members, North Holland Publishing Company, 1969

[27] J. Lemaitre, J.L. Chaboche, A non-linear model of creepfatigue damage cumulation and interaction, Symposium IUTAM sur la mécanique des milieux et des corps viscoélastiques, Gothenburg, Suède, septembre 1974

[28] J. Lemaitre, A course on damage mechanics, SpringerVerlag, Berlin, 1995

[29] J. Lemaitre, A continuous damage mechanics model for ductile fracture, J. Engng. Mat. Tech. 107 (1985) 83-89

[30] J. Lemaitre, J.P. Sermage, R. Desmorat, A two scale damage concept applied to fatigue, Int. J. Frac. 97 (1999) $67-81$

[31] J.L. Chaboche, Une loi différentielle d'endommagement de fatigue avec cumulation non linéaire, Revue Française de Mécanique (50-51) (1974) 71-82

[32] J.L. Chaboche, La fatigue des matériaux et des structures, Hermès, 1997, pp. 617-644

[33] S. Taira, Relationship between thermal fatigue and lowcycle fatigue at elevated temperature, in : Fatigue at Elevated Temperature - ASTM STP 520, 1973, pp. 20101

[34] J.L. Chaboche, F. Gallerneau, An overview of the damage approach to durability modeling at elevated temperature, Fatigue Fract. Engng. Mater. Struct. 24 (6) (2001) 405417

[35] A. Bignonnet, Fatigue design in automotive industry, in : G. Lutjering, H. Nowack (Eds.), Fatigue'96 - 6th International Fatigue Congress, 1996, pp. 1825-1836
[36] L. Remy, Thermal and thermal-mechanical fatigue of superalloys - a challenging goal for mechanical tests and models, in : K.T. Rie, P.D. Portella (Eds.), Low Cycle Fatigue and Elasto-Plastic Behaviour of Materials, Elsevier, 1998, pp. 119-130

[37] L. Verger, A. Constantinescu, E. Charkaluk, Thermomechanical fatigue design of aluminium components, in : Fatigue-Temperature Interaction, 9th International Spring Meeting of the SF2M, May 2001, pp. 293-302

[38] B. Halphen, Q.S. Nguyen, Sur les matériaux standards généralisés, J. Mécanique 14 (1) (1975) 39-631

[39] E. Charkaluk, Dimensionnement des structures à la fatigue thermomécanique, Ph.D. thesis, spécialité : mécanique, École Polytechnique, France, 1999

[40] L. Verger, A. Constantinescu, E. Charkaluk, On the simulation of large viscoplastic structures under anisothermal loadings, in : Murakami and Ohno (Eds.), IUTAM Creep in Structures, Kluwer, April 2000

[41] L. Bourgeois, Contrôle optimal et problèmes inverses en plasticité, Ph.D. thesis, spécialité : mécanique, École polytechnique, France, 1998

[42] J.C. Simo, T.J.R. Hughes, Computational inelasticity, Springer-Verlag, 1998

[43] A. Aouameur, E. Charkaluk, Influence du modèle d'écrouissage sur la réponse cyclique anisotherme d'une structure $3 \mathrm{~d}$, in : CSMA (Ed.), Actes du $5^{\mathrm{e}}$ colloque national en calcul des structures, Teknea, 2001, pp. 343-350

[44] H. Maïtournam, B. Pommier, J.J. Thomas, Détermination de la réponse asymptotique d'une structure anélastique sous chargement thermomécanique cyclique, C.R. Mécanique 330 (2002) 703-708

[45] G.R. Halford, S.S. Manson, Life prediction of thermalmechanical fatigue using strainrange partitioning, in : Thermal Fatigue of Materials and Components - ASTM STP 612, 1976

[46] J.L. Chaboche, H. Policella, H. Kaczmarek, Applicability of the srp method and creep-fatigue approach to the lchtf life prediction of in-100 alloy, in : réunion AGARD sur la caractérisation de la fatigue oligocyclique à chaud par la méthode de partition de l'amplitude de déformation, Aalborg, Danemark, avril 1978

[47] R.P. Skelton, Energy criterion for high temperature low cycle fatigue, Mat. Sci. Tech. 7 (1991) 427-439

[48] R.P. Skelton, T. Vilhelmsen, G.A. Webster, Energy criteria and cumulative damage during fatigue crack growth, Int. J. Fatigue 20 (9) (1998) 641-649

[49] G. Marquis, D. Socie, Long-life torsion fatigue with normal mean stresses, Fat. Frac. Engng. Mat. Struct. 23 (2000) 293-300 